\title{
Calidad en el servicio en micronegocios del sector artesanal de madera en una comisaría de Mérida, México
}

\author{
Quality service in micro business in the wood crafts \\ sector in a suburb of Mérida, México \\ Marbella Guatzozón Maldonado ${ }^{1 *} \quad$ Ana María Canto Esquivel ${ }^{1} \quad$ Andrés Pereyra Chan $^{1}$ \\ Recibido 06 de mayo de 2018, Aceptado 08 de octubre de 2018 \\ Received: May 06, 2018 Accepted: October 10, 2018
}

\begin{abstract}
RESUMEN
Los micronegocios tienen particular importancia para las economías nacionales, no solo por sus aportaciones a la producción y distribución de bienes y servicios, sino también por el gran potencial de generación de empleos. Representan un excelente medio para impulsar el desarrollo económico y una mejor distribución de la riqueza. México, como otros países del mundo, tiene como eje vertebral económico a los micronegocios. En el Estado de Yucatán también los micronegocios son cruciales para el desarrollo. Sin embargo, éstos se enfrentan cada vez -con mayores dificultades debido a la competencia y los mercados cambiantes. Complicando así su permanencia en el mercado y provocando su cierre. Por ello, se ha empleado la calidad en el servicio como herramienta para potencializar la competitividad, de forma que permita la supervivencia de estos micronegocios. En el presente estudio se analiza específicamente a micronegocios de artesanías de madera en una comisaría de Mérida, Yucatán. Esto cobra particular importancia ya que las artesanías son patrimonio cultural de la nación. La metodología es de enfoque cuantitativo, no experimental, de corte transversal y descriptiva, determinando diferencias entre expectativas y percepciones de los clientes con base en la calidad en el servicio de 10 micronegocios, localizados en un estudio de campo y que aceptaron participar en el estudio, aplicando una adaptación del instrumento Servqual de Zeithaml, Parasuraman y Berry [1], con 19 ítems y escala Likert de 7 puntos. Finalmente, se discuten los resultados obtenidos y se realizan propuestas para el diseño de estrategias que permitan mejorar la calidad del servicio y la permanencia en el mercado.
\end{abstract}

Palabras clave: Calidad en el servicio, micronegocios, madera.

\section{ABSTRACT}

The micro businesses are particularly important for national economies, not only because of their contributions to the production and distribution of goods and services, but also because of the high potential for job creation. They represent an excellent means to boost economic development and a better distribution of wealth. Mexico, like other countries in the world, has micro-business as an economic backbone. In the State of Yucatan, micro-businesses are also crucial for development. However, they face increasing difficulties due to competition and changing markets. Complicating its permanence in the market and causing its closure. For this reason, quality in the service has been used as a tool to boost competitiveness, in a way that allows the survival of these micro-businesses. This analysis is particularly

\footnotetext{
1 Tecnológico Nacional de México. Instituto Tecnológico de Mérida. Departamento de Ingeniería Industrial. Dirección Km.5, Carretera Mérida - Progreso A.P. 911. C.P. 97118. Mérida. Yucatán, México.

E-mail: amc.esquivel@gmail.com; pereyraandres@hotmail.com; marbella_gmaldonado@ hotmail.com

* Autor de correspondencia: marbella_gmaldonado@hotmail.com
} 
important since the crafts are the cultural heritage of the nation. The methodology is a quantitative approach, not experimental, cross-sectional and descriptive, determining differences between expectations and perceptions of clients based on the quality in the service of 10 micro-businesses, located in a field study and who agreed to participate in the study. , applying an adaptation of the SERVQUAL instrument of Zeithaml, Parasuraman and Berry [1], with 19 items and a Likert scale of 7 points. Finally, the obtained results are discussed, and proposals are made for designing strategies to improve the quality of the service and the permanence in the market.

Keywords: Quality in the service, micro-business, wood.

\section{INTRODUCCIÓN}

Todos los días en el mundo, se hace uso de una amplia gama de servicios de manera tan rutinaria que no se repara en ellos; como servicios en los supermercados, tiendas departamentales, restaurantes, hoteles, hospital, cinemas, gasolineras, etc. En todas estas acciones se hace uso de los servicios y por desgracia no siempre la calidad es satisfactoria para los clientes. Es probable que, en ocasiones, las experiencias de servicio que tengan los clientes sean con una buena percepción y satisfactoria pero habrá otras en las que sean muy decepcionantes llevándose malas experiencias.

Las micro y pequeñas empresas ocupan un lugar importante dentro de la economía de México, son fuentes de empleo de muchas familias y son de gran importancia como generadoras de PIB nacional, por lo que buscar estrategias para que compitan en el mercado es de gran relevancia para la estabilidad de la economía del país. En los micronegocios de artesanías de madera en la comisaría de Mérida, se ven en la necesidad de competir día con día debido a la apertura de otros negocios o debido a que los clientes visitan otros establecimientos comerciales como tiendas de artesanías, lugares turísticos y hasta en las mismas carpinterías que se encuentran en las poblaciones vecinas y principalmente en los establecimientos en la ciudad de Mérida, por las razones ya comentadas anteriormente, mejorar la calidad en el servicio sería una ventaja competitiva y así ayudar a que puedan sobrevivir en el mercado actual.

Bajo el enfoque del cliente, si un negocio puede hacer que su producto o servicio sea especialmente atractivo, sus posibilidades se ampliarán de manera considerable. Un buen servicio al consumidor lo puede ofrecer un negocio de cualquier clase, sin embargo la MiPyME tienen un potencial más preciso que las empresas de mayor tamaño para alcanzar esta meta. Si se maneja adecuadamente, la MiPyME pueden atender a los clientes sin luchar a través de niveles de burocracia o romper políticas corporativas que tienden a frenar la iniciativa del personal [2].

Los micronegocios de la comisaría de Mérida en el estado de Yucatán, no son ajenos a las afectaciones de los mercados cambiantes. En particular el presente estudio está enfocado a los micronegocios de artesanías de madera para ofrecer beneficios para desarrollarse en el mercado y que sean suficientemente competentes. Las necesidades de los micronegocios de artesanías de madera son varias. Esto hace que tengan que competir en mercados más complejos y cambiantes.

Los micronegocios deben capacitar y motivar eficazmente a su personal de contacto con los clientes y a todos los empleados de apoyo de servicio, para que trabajen como un equipo y proporcionen satisfacción al cliente. Todos en el micronegocio deben tener una orientación hacia el cliente. La importancia de ofrecer bienes y servicios de calidad que logren la satisfacción total de los clientes es una necesidad imperiosa en un mundo en donde la competencia es cada vez más feroz y el consumidor se ha vuelto más exigente en la búsqueda de una mejor calidad.

Es un hecho que, todos los días se crean nuevas empresas, pero el reto está en su permanencia en el mercado y conseguir que éstas crezcan. Es por eso que, el presente trabajo estudia la posibilidad de que la calidad en el servicio pueda ser tomada como una ventaja competitiva para los micronegocios de artesanías de madera en una comisaría de Mérida, Yucatán. 
El sector artesanal se encuentra inmerso dentro de la clasificación de MiPyMEs, cuya relevancia económica reside en que constituyen el 99,8\% de las empresas en México [3]. Las organizaciones de este tipo se encuentran a lo largo y ancho del país y del mundo, constituyendo un factor importante del sistema económico, tanto por su contribución al empleo como por su aportación al Producto Interno Bruto (PIB).

Las MiPyMEs, artesanales, en el contexto de la globalización, se convierten en una alternativa válida para ciertos grupos sociales, en donde se genera empleo y se aprovecha el potencial creador y artístico que desde la época prehispánica han tenido los pueblos indígenas. Es una oportunidad para proponer estrategias y que mejor que la calidad en el servicio, con el propósito de que mejoren su situación económica, social y puedan prevalecer en el mercado [3].

Existen estudios en otros países y publicaciones en otros estados de la República Mexicana sobre calidad en el servicio en el sector comercio al por menor, los cuales se abordarán más adelante en esta investigación. Esta investigación servirá para el desarrollo de los micronegocios de artesanías de madera en el Estado de Yucatán a través de conocer el grado de calidad en el servicio que tienen los clientes en ese tipo de negocios.

El presente trabajo se enfocará a analizar las expectativas y percepciones de la calidad en el servicio de los clientes de los micronegocios de artesanías de madera en una comisaría de Mérida, Yucatán, con él se pretende definir qué factores consideran los usuarios determinantes en un servicio de calidad y así enfocarse en la mejora continua y de la seguridad que se le brinda a los clientes, además de impulsar a otros micronegocios a mantener sus ventajas competitivas para alcanzar, sostener y mejorar su posición en el entorno y dirigir esfuerzos para lograr la satisfacción del usuario.

\section{ANÁLISIS TEÓRICO}

\section{Calidad en el servicio}

En la investigación de la calidad del servicio, es el enfoque más ampliamente aceptado se basa en la premisa de que la evaluación que realiza el cliente sobre la calidad de algún servicio depende de la magnitud y la dirección de la brecha entre sus expectativas sobre el servicio y la evaluación (percepción) del servicio que realmente reciba [4].

La calidad percibida se da cuando los clientes juzgan la calidad de un producto o servicio con base en una variedad de señales informativas que asocian con el producto; algunas de estas señales son intrínsecas al producto o servicio; otras son extrínsecas, como el precio, la imagen de la tienda, el entorno del servicio, la imagen de la marca y los mensajes promocionales [4].

En el ámbito mundial y en el contexto de la globalización, la calidad del servicio cada día cobra mayor relevancia pues si se maneja en forma adecuada hace que las organizaciones logren ventajas competitivas que les generan un valor agregado incalculable para las mismas [5].

Es más difícil evaluar por parte de los clientes la calidad en el servicio, debido a que las características son intangibles, variables, perecederas y se consumen en el mismo momento en que se producen. Los clientes confían en el precio como un indicador de calidad y atribuyen calidades diferentes a productos que realmente son idénticos pero que difieren en los precios; la percepción de valor también se ve influenciada por aspectos como la edad y los niveles de ingreso [4].

La calidad en el servicio es el hábito desarrollado y practicado por una empresa para interpretar las necesidades y expectativas de sus consumidores y ofrecerles, en consecuencia, un servicio accesible, adecuado, ágil, flexible, apreciable, útil, oportuno, seguro y confiable, aún bajo situaciones imprevistas o ante errores, de tal manera que el cliente se sienta comprendido, atendido y servido personalmente, con dedicación y eficacia, y con mayor valor al esperado, proporcionando así a la empresa la estabilidad o como herramienta para obtener ventajas competitivas y ganar la fidelidad de los clientes [6].

Los bienes intangibles se denominan, generalmente, servicios. Su estructura es inmaterial. Se trata de actos que recibe el cliente y a través de los cuales soluciona sus problemas o carencias. En general, se puede entender por producto tanto un tangible como un intangible, siempre que esté referido a la prestación principal de la organización y, más concretamente, del departamento o unidad [7]. 
El cliente percibe como valioso, además de la prestación principal, una serie de elementos adicionales: 1) las prestaciones añadidas a la principal, 2) el modo de entrega de la prestación principal y 3) la combinación de ambas. Sobre lo anterior está la conceptualización de servicio como el conjunto de prestaciones accesorias que acompaña a la prestación de la principal [7].

La calidad en el servicio ha evolucionado por lo que se ha vuelto importante, ya que la economía global se convirtió en una economía de servicios donde la participación del sector servicios es elevada y generando empleos que crea la economía [1]. El servicio constituye una parte importante de todo lo que, se produce, se consume y se exporta ya que tiene una gran participación en las organizaciones.

La calidad en el servicio consiste en prestar atención y cuando se hace esto las organizaciones se convierten más eficientes ya que se cumplen con los requisitos indispensables para serlo: preguntar y escuchar. La calidad en el servicio no es conformidad con las especificaciones, sino más bien conformidad con las especificaciones de los clientes. La calidad en el servicio adquiere realidad en la percepción, considerando esta como un deseo más que la percepción, ya que esta última implicaría un pensamiento y análisis anterior [8].

\section{La importancia de la calidad en el servicio en el cliente}

El servicio al cliente es el servicio o atención que una empresa o negocio le brinda a sus clientes al momento de ofrecer productos y servicios correctos además de responder preguntas, dar solución a problemas en el momento adecuado y así dar un seguimiento continuo creando una fidelidad mayor. La calidad del servicio al cliente es una ventaja importante porque puede hacer la diferencia en cualquier empresa o negocio. El impacto que éste tiene puede ser la razón por la cual los clientes se inclinan por determinada marca, es decir, cualquier paso equivocado que realice la empresa o negocio llevará al cliente directamente hacia sus competidores directos. Los clientes anteriormente buscaban que los productos o servicios se ajustaran a sus necesidades solo en calidad y un buen precio, pero a través de los años el cambio fue evidente, el servicio al cliente fue trascendental para crear fidelidad entre el cliente y las empresa [9].
La calidad en el servicio es uno de los aspectos importantes para asegurar la permanencia de las empresas en el mercado, sin embargo hoy nos encontramos con gran número de clientes disconformes principalmente con los servicios que se le entregan. Lo verdaderamente importante es poder medir los niveles de satisfacción con la finalidad de poder obtener datos que le ayuden a la empresa a tomar decisiones orientadas a mejorar la calidad de servicio y de esta forma conseguir un elemento que cree una ventaja por sobre sus competidores [10].

\section{Estudios empíricos de calidad en el servicio}

En diversos países se han realizado estudios empíricos de la calidad en el servicio, contribuyendo a diversas organizaciones para obtener la fidelidad de sus clientes haciendo que estas sean competitivas en el mercado en el que se encuentren.

En Venezuela se determinó la calidad del servicio en un restaurante de comida rápida, la investigación fue descriptiva abordándose a través de un diseño de campo, los resultados obtenidos mediante la aplicación del modelo Servqual fue que existieron déficit de calidad en los indicadores [11].

Se ha realizado revisiones bibliográficas respecto a algunos de los modelos de evaluación más representativos en la literatura científica, se caracterizan y analizan considerando sus perspectivas, dimensionalidad, aplicación y alcance [12].Se ha observado que la percepción del cliente es la perspectiva más utilizada en estas evaluaciones, destacándose el uso de modelos multidimensionales, basados en indicadores externos, donde la tangibilidad, atención al usuario, fiabilidad, seguridad, capacidad de respuesta y resultados son las dimensiones de evaluación más utilizadas. El modelo Servqual es considerado un referente importante, siendo citado, analizado y aplicado en numerosas y recientes investigaciones, en diversos tipos de servicios [12] .

Se ha realizado análisis de la validez y confiabilidad del instrumento de medición de la calidad del servicio mediante los modelos de Servqual y Servperf aplicado en cafeterías universitarias. Consistió en validar una escala, de 19 ítems que fue utilizado en las universidades públicas para evaluar la calidad del servicio percibido por los usuarios; para lograrlo se realizó un estudio en una muestra de 297 usuarios, en el cual se evaluaron las propiedades de una 
escala integrada por tres variables. Los resultados revelan la existencia de tres variables que explican el $63.186 \%$ de la variación. Las pruebas de validez y consistencia interna de la escala son satisfactorias. Se afirmó que el instrumento es válido y útil para evaluar la calidad de los servicios de las cafeterías en el campus en donde se realizó la investigación [13].

El análisis de percepción de calidad en los consumidores de la industria hotelera es un tema que ha interesado mucho al marketing, ya que en este tipo de servicios se considera de alta relevancia el logro de la satisfacción del cliente para concretar la fidelidad de los clientes, la realización del trabajo tuvo como finalidad revisar conceptos sobre la calidad en el servicio hotelero no solo a nivel teórico sino de estudios empíricos realizados por muchos estudiosos en el tema. Además, se encuentra que existe una amplia variedad de modelos ideados para medir la satisfacción de los clientes como el Servqual y el Hotelqual y que aún no hay un consenso sobre las variables por analizar en el momento de medir las percepciones de un cliente quien, después de tomar el servicio puede estar satisfecho o insatisfecho [14].

En los hoteles de Resort de bahías de Huatulco en el Estado de Oaxaca, la escala Hotelqual fue empleada para el trabajo de campo ya que es un instrumento confiable y práctico que supera las deficiencias del modelo Servqual. Al medir la calidad del servicio se obtienen valoraciones globales superiores a la media. La dimensión Instalaciones es la que más sobresale. Los aspectos tangibles tienen un fuerte impacto en la percepción y satisfacción del turista en los servicios hoteleros [15].

Varela, Prat, Voces y Rial [16] aplicaron un instrumento al servicio de la hostelería en el área de la restauración en Santiago de Compostela y alrededores, considerando cinco dimensiones analizadas: acceso, personal, servicio, producto e instalaciones. Como resultado tuvo mayor importancia el producto, luego el personal, instalaciones, acceso y definieron eliminar la dimensión servicio, escala propuesta para evaluar la calidad percibida.

\section{Servqual}

Servqual fue diseñado por Zeithaml, Parasuraman y Berry [1] y es un instrumento de 22 ítems que permite medir las expectativas y percepciones de los usuarios respecto a los cinco criterios sobre la calidad: 1) elementos tangibles los cuales son apariencia de las instalaciones físicas, equipos, personal y materiales de comunicación; 2) fiabilidad que conforma la habilidad para realizar el servicio prometido de forma fiable y cuidadosa; 3 ) capacidad de respuesta que es la disposición y voluntad para ayudar a los usuarios y proporcionar un servicio rápido; 4) la seguridad son los conocimientos y atenciones mostrados por los empleados y sus habilidades para inspirar credibilidad y confianza y 5) empatía es la atención individualizada que ofrecen las empresas a sus clientes.

El modelo Servqual de calidad en el servicio permite disponer de puntuaciones sobre percepción y expectativas respecto a cada característica del servicio evaluada. La diferencia entre percepción y expectativas indicará los déficits de calidad cuando la puntuación de expectativas supere a la de percepción [17].

$\mathrm{Al}$ aplicarse Servqual a muestras de clientes mide: una calificación global de la calidad del establecimiento, lo que desean los clientes de ese establecimiento, lo que encuentran los clientes en ese establecimiento, calcula brechas de insatisfacción específicas y ordena defectos de calidad desde el más grave y urgente hasta el menos grave [18].

Servqual, centra su atención en el proceso de entrega del servicio más que en el resultado de la provisión del servicio, es decir del encuentro del proveedor con el consumidor. En la actualidad Servqual es el modelo de investigación más usado para medir la calidad en la industria de servicios [18].

En esta investigación se consideró lo más adecuado adaptar el modelo de Servqual, desarrollado por Zeithaml, Parasuraman y Berry [1] para medir la calidad en el servicio usando dos cuestionarios correspondiente a las expectativas y percepciones de 19 ítems, modificado para el cumplimiento del objetivo de esta investigación.

\section{ANÁLISIS CONTEXTUAL}

\section{Micronegocios en México}

Un micronegocio es una empresa de tamaño pequeño. La definición de esta varía de acuerdo a cada país, aunque, generalmente, puede decirse que una microempresa cuenta con un máximo de diez 
empleados y su facturación es acotada. Por otra parte, cabe señalar que la mayoría de los dueños de los micronegocios son los que, personalmente se encargan de trabajar en ellas debido al tamaño pequeño de la empresa [19].

En México muchas personas comienzan micronegocios porque no encontraron empleo como trabajadores asalariados, porque fueron despedidos, o porque su situación familiar no les permitió tomar un trabajo de tiempo completo con un horario fijo [20]. En otras palabras, empezar un negocio es una segunda opción que pueden percibir como temporal, mientras encuentran un trabajo o cambia su situación familiar, esos microempresarios probablemente no tienen grandes planes de expansión ni están dispuestos a invertir mucho. La gran mayoría de las empresas en México son de menos de cinco trabajadores. Muchas parecen ser formas de autoempleo o situaciones transitorias. Otras parecen ofrecer al dueño flexibilidad e independencia.

Las unidades económicas del país presentaron una tendencia positiva en el número de establecimientos y personal ocupado. En el periodo 2003-2008, los establecimientos del país crecieron $23,9 \%$, mismo porcentaje que el personal ocupado total; mientras que de 2008 a 2013, el crecimiento fue de 13,6\% y en el personal ocupado de 7,3\%. Durante los años de 2003 a 2013 se registró una variación de 40,8\% en establecimientos y $32,9 \%$ en personal ocupado [21].

$\mathrm{Al}$ analizar la estructura porcentual de las unidades económicas y el personal ocupado por estratos, se observó que no ha sufrido cambios significativos en los últimos 10 años. Es notoria la participación de los establecimientos que ocupaban hasta 10 personas, pues mientras que sus porcentajes de participación fueron de 94,9, 95,0 y 95,4\% para 2003, 2008 y 2013, respectivamente, el personal ocupado en estas unidades representó 38,3, 41,8 y $39,8 \%$ para esos años [21].

Los establecimientos micro en el país, que agrupan a todas aquellas unidades que ocuparon hasta 10 personas, representaron $95,3 \%$ del total de las unidades económicas prestadoras de Servicios en México, ocupando el 43,1\% del personal ocupado total, las remuneraciones de este tamaño representaron $12 \%$ los ingresos, $22,4 \%$ y los activos fijos, $24,3 \%$ [22].
Las MiPyMEs, son muy importantes para la economía de cualquier país. Tan sólo en México son más de cuatro millones, generan $72 \%$ del empleo y aportan $52 \%$ del Producto Interno Bruto (PIB). Estos negocios son los que hacen competitivo a un país, los que atraen inversiones y fortalecen las industrias. Sin embargo, la incertidumbre económica en el mundo ha hecho que algunas empresas en desarrollo no se consoliden apropiadamente y sean vulnerables ante los constantes cambios del mercado [23].

Las MiPyMEs de México han comenzado a buscar formas innovadoras para crecer, y una de sus principales estrategias es tratar de incursionar en nuevos mercados, tanto nacionales como internacionales [23].

Situación actual de los micronegocios de Yucatán El estado de Yucatán, está ubicado en el sureste de la República Mexicana, colinda al norte con el Golfo de México, al sureste con el estado de Quintana Roo y al suroeste con el estado de Campeche. Es uno de los estados del país con mejor ubicación geográfica y mayor facilidad de acceso. Su posición geográfica es privilegiada.

Según datos del Censo Económico 2014, el estado de Yucatán reportó la cantidad de ingresos de 261,554 millones de pesos, que representan $1,5 \%$ de los ingresos del país. El municipio de Yucatán más dinámico fue el Mérida con 205,879 millones de pesos en ingresos, lo que representó $78,7 \%$ del total estatal. De ahí siguió el municipio de Umán con una participación de 7,8\% en los ingresos generados en la entidad. La participación en los ingresos de los establecimientos que ocuparon hasta 10 personas representa el 19\% del total estatal siendo estos los micronegocios [24].

Yucatán cuenta con un total de 98,478 unidades económicas, de las cuales el 39,28\% corresponde al sector de comercio; el $34,75 \%$ a servicios y $22,98 \%$, a la industria manufacturera; el restante $2,98 \%$ corresponde a los otros [25]. De los 98,478 unidades económicas que hay, el 94177 lo ocupa los micronegocios con un personal ocupado de 201635, las pequeñas empresas son 3543 con personal ocupado 71898, las empresas medianas 652 y de personal 69007 y por último, las grandes empresas en Yucatán son 106 con personal ocupado de 79421 [25]. Es claro mencionar que los micronegocios son el principal sustento del estado. 


\section{Panorama local de artesanías en Yucatán}

La actividad artesanal, sigue estando vigente en pleno siglo XXI, porque en todo el mundo se producen diversos productos y artículos artesanales, originados en culturas ancestrales, que contrastan la mayoría de las veces con los producidos por la revolución tecnológica y los avances en diversas áreas del conocimiento; además, las situaciones y divisiones socioeconómicas aún prevalecen para esta actividad económica [25].

Yucatán es uno de los estados que son conocidos por sus raíces en la cultura maya cuenta con una producción artesanal de gran calidad y diversidad [26]. En todo Yucatán se elaboran artículos de los más diversos materiales, diseños y colores, que además de ser útiles, aportan belleza y elegancia al entorno: joyería de filigrana en oro y plata; artefactos de carey, concha, caracol, madera y piedra tallada; vasijas de barro; instrumentos musicales; tejidos de fibra de henequén y de algodón [27].

En Yucatán, el tallado en madera es una actividad que se remonta a la época prehispánica, pues los antiguos mayas trabajaban la madera para proveerse de instrumentos de trabajo, musicales, objetos ceremoniales y mobiliario. Se puede decir que el tallado de madera aún persiste, se realiza de manera artesanal con fines comerciales en tres grandes áreas: el sur, el poniente y el oriente de Yucatán. En el área sur se incluye la villa de Muna y localidades aledañas a los sitios arqueológicos que comprenden la Ruta Puuc; en el poniente está Dzityá que es comisaría de la ciudad de Mérida; y en el oriente, se encuentra Pisté, Popolá, Cuncunul, Tixhualahtún y Chemáx [27].

\section{Comisaría de Dzityá, Yucatán}

Ubicada al norte de la ciudad capital a una distancia de 10 minutos, cuenta actualmente con dos accesos, uno por la carretera Mérida - Progreso y otro por el fraccionamiento Francisco de Montejo [26]. Dzityá cuenta con todos los servicios urbanos siendo su principal actividad económica la artesanía.

La población total de Dzityá es de 1496 personas, de los cuales 766 son masculinos y 730 femeninas. Los ciudadanos se dividen en 558 menores de edad y 938 adultos, de ellos 93 tienen más de 60 años. 669 personas en Dzityá viven en hogares indígenas. Su idioma indígena es Maya [28].
Este pueblo se caracteriza por ser creadores de piezas y figuras que atrapan la atención. En este lugar los antepasados usaban tornos de pedal con los cuales únicamente se podían elaborar molcajetes y batidoras; actualmente la producción se ha diversificado y el modelo del trabajo también. Entre los principales productos de artesanía se encuentra: fruteros, floreros, tortilleros, candelabros, salseros, alhajeros, bases, azucareras, chileros, entre otros [29].

Guatzozón, Canto y Ulibarri [30], realizaron un recorrido por Dzityá donde localizaron 49 micronegocios. Estos se pueden dividir en tres categorías: 13 micronegocios son de artesanías de madera, 17 son artesanías piedra y otros que engloba a los abarrotes, molinos, panaderías y papelería son 19 . Cabe aclarar que se reconoce la importancia que tienen las artesanías en la economía de Dzityá, ya que ha sido desde sus inicios un lugar de artesanos.

\section{METODOLOGÍA}

En este apartado, se presenta la metodología que sirvió de guía para realizar la investigación. El enfoque del estudio es cuantitativo, ya que se usó la recolección de datos para la medición numérica y el análisis estadístico, con el fin de cumplir el objetivo de la investigación, evaluando las expectativas y percepciones de los clientes en la calidad en el servicio de los micronegocios de artesanías de madera en la comisaría de Mérida, mediante las cinco dimensiones de Zeithaml, Parasuraman y Berry [1].

La investigación es no experimental, el alcance fue descriptivo ya que se estableció el nivel de calidad en el servicio usando las expectativas y percepciones de los clientes de los micronegocios de artesanías de madera en la comisaría de Mérida, Yucatán. Su temporalidad fue transversal ya que se recolectaron datos en un solo momento.

La unidad de análisis fueron los micronegocios de artesanías de madera de la comisaría de Dzityá del municipio de Mérida, Yucatán y los sujetos de estudio fueron los clientes, ya que a ellos se les aplicaron los instrumentos de estudio para poder analizar expectativas y percepciones sobre la calidad en el servicio ofrecida en los micronegocios.

Guatzozón, Canto y Ulibarri [30], en su estudio localizaron 13 micronegocios de artesanías de 
madera mencionado anteriormente, de lo cual 10 aceptaron participar en el estudio por lo que se hizo una entrevista a los dueños para que proporcionaran la cantidad de clientes y como resultado se obtuvo 336 clientes conformando la población de estudio. Para seleccionar el tamaño de la muestra se aplicó la fórmula de Castañeda, Torre, Morán y Lara [31], ya que se conoce el tamaño de la población de los clientes, la cual brindó una muestra de 178 clientes. La ecuación (1), de acuerdo con estos autores es la siguiente:

$$
n=\frac{z^{2} p q N}{N e^{2}+Z^{2} p q}
$$

Donde: $n=$ tamaño de muestra, $Z^{2}=$ nivel de confianza, $p=$ variabilidad positiva, $q=$ variabilidad negativa, $e^{2}=$ error, $N=$ tamaño de población.

Se utilizó un nivel de confianza de 1,96, una variabilidad de 0,5 y un error estimado de $5 \%$. La muestra fue no probabilística, pues se incluyó en ella a 10 micronegocios de artesanías de madera que aceptaron participar en el estudio, se aplicaron 178 encuestas a los clientes que cumplieron con los requisitos establecidos para participar y que además desearon hacerlo. Cabe recalcar que la aplicación de las encuestas era variado, debido a la afluencia de clientes de cada negocio, en función de esto, se calculó el promedio de encuestas aplicadas tal que mientras en unos se aplicaron solo 6 encuestas, en los de mayor clientela se aplicaron 32 .

El instrumento de recolección que se utilizó en la investigación, fue la adaptación del instrumento SERVQUAL hecha para los micronegocios de artesanías de madera en la comisaría de Mérida, Yucatán [1]. El instrumento permite cuantificar la calidad en el servicio evaluando por separado las expectativas y percepciones de los clientes mediante dos cuestionarios de 19 items cada uno. La escala de medición se basa en cinco dimensiones que los autores distinguen: Elementos tangibles, Confiabilidad, Capacidad de respuesta, Responsabilidad, Empatía.

En los cuestionarios de expectativas y percepciones se contó con reactivos que incluyen la medición de las cinco dimensiones de la calidad ya mencionadas; ambas secciones se evaluaron a través de una escala Likert de 7 puntos, donde 1 es Totalmente en desacuerdo y 7 es Totalmente de acuerdo.
En la medición de la calidad del servicio, fue necesario calcular la diferencia entre la percepción del cliente y el ideal del servicio, lo cual se hizo el análisis de la información obtenida. Se utilizó SPSS que es un programa computacional especializado en el análisis.

\section{RESULTADOS}

Se presenta a continuación los resultados de la aplicación de los instrumentos de investigación para el cumplimiento del objetivo.

\section{Expectativas de los clientes sobre la calidad en el servicio de los micronegocios de artesanías de madera}

De las 178 encuestas aplicadas a los clientes de 10 micronegocios de artesanías de madera, se obtuvo puntajes de las expectativas de cada dimensión de la calidad en el servicio de los clientes, la dimensión de empatía obtuvo el mayor puntaje de 6,46, siendo ésta la más importante por los clientes, debido a que la atención individualizada ya que es importante para ellos; la dimensión de seguridad con 6,42 puntos es la que ocupa el segundo lugar debido a que para los clientes el conocimiento de los empleados es primordial para la credibilidad y confianza de los micronegocios de artesanías de madera; la tercera dimensión es la capacidad de respuesta que obtuvo 6,40 puntos con la finalidad de que los empleados tengan disponibilidad y voluntad para ayudar a los clientes y poder proporcionarles un servicio rápido; la cuarta dimensión es fiabilidad con 6,38 puntos ya que los clientes esperan que el servicio brindado sea cumplido de manera fiable y cuidadoso; y con menor importancia fue la dimensión de elementos tangibles con 6,14 puntos de expectativas en la calidad en el servicio por parte de los clientes debido a que en la apariencia física de las instalaciones, equipos y personal, no es para ellos de gran importancia a comparación con las otras cuatro dimensiones.

\section{Percepciones de los clientes sobre la calidad en el servicio de los micronegocios de artesanías de madera}

En las 178 encuestas aplicadas a los clientes de 10 micronegocios de artesanías de madera, se obtuvo puntajes en percepciones de cada una de las cinco dimensiones de la calidad en el servicio de los clientes de los micronegocios de artesanías de madera, fueron evaluados por los clientes y como resultado 
la dimensión de seguridad obtuvo el mayor puntaje con 5,64 puntos ya que en algunos establecimientos tenían conocimientos de las artesanías y mostraban interés a sus clientes, siguiendo la dimensión de fiabilidad con 5,434 puntos ya que no en todos los micronegocios cumplían con las entregas en la fecha acordada, la siguiente dimensión es capacidad de respuesta con 5,430 puntos a causa de que el personal de los micronegocios no tenían disposición y voluntad para ayudar a los clientes y proporcionarles servicio rápido, la cuarta dimensión es empatía con 5,30 puntos debido a que los clientes no recibían atención individualizada por parte de los empleados y con menor calificación en percepción en la calidad en el servicio fue la dimensión de elementos tangibles con 5,12 puntos, debido a que en los micronegocios no tienen equipos modernos como lo son vitrinas, estantes o equipo de cómputo para registrar las compras, al igual que en las fachadas no están en buenas condiciones y dentro de los establecimientos no contaban con buena iluminación y ventilación para apreciar las artesanías de madera.

\section{Calidad en el servicio de acuerdo a la diferencia entre expectativas y percepciones de los clientes} de los micronegocios de artesanías de madera

En la Tabla 1, se muestra las puntuaciones por dimensión a lo que respecta las expectativas y percepciones, de ahí la diferencia entre ellas que es la brecha donde se observa que todas son negativas, ya que ninguna de las dimensiones cubre las expectativas de los clientes; en la última columna es representado de manera general la calificación porcentual que obtuvo cada dimensión. Se puede decir que las puntuaciones globales de las dimensiones en expectativas se obtuvo 6,36 puntos y las percepciones 5,38 puntos teniendo como brecha $-0,97$ puntos de diferencia por lo que existe insatisfacción por parte de los clientes debido a que sus expectativas esperadas por parte de los micronegocios de artesanías de madera no fueron cumplidas teniendo una percepción inferior a la de expectativas, por lo que es necesario realizar propuestas de mejora para que los clientes estén satisfechos y poder cumplir con sus expectativas o incluso superarlas. La dimensión de seguridad obtuvo la mayor calificación con $88 \%$, siguiendo la fiabilidad y capacidad de respuesta con $85 \%$ ambos con la misma calificación porcentual debida a la mínima diferencia entre ellos, con $83 \%$ los elementos tangibles y con la menor calificación fue empatía con una calificación porcentual de $82 \%$. Por último, cabe destacar que el $85 \%$ es la calificación general de la calidad en el servicio que brindaron los micronegocios de artesanías de madera hacia sus clientes, por lo que es necesario propuestas de mejora para la fidelidad de los clientes y la estabilidad en el mercado.

Tabla 1. Puntuaciones Globales de las cinco dimensiones en los micronegocios de artesanías de madera.

\begin{tabular}{|c|c|c|c|c|}
\hline 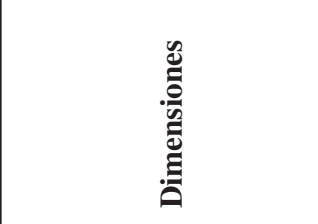 & 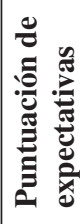 & 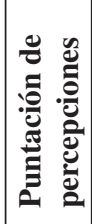 & 吾 & 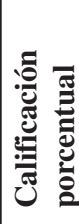 \\
\hline Elementos Tangibles & 6,14 & 5,12 & $-1,01$ & $83 \%$ \\
\hline Fiabilidad & 6,38 & 5,434 & $-0,94$ & $85 \%$ \\
\hline Capacidad de Respuesta & 6,40 & 5,430 & $-0,97$ & $85 \%$ \\
\hline Seguridad & 6,42 & 5,63 & $-0,78$ & $88 \%$ \\
\hline Empatía & 6,46 & 5,29 & $-1,16$ & $82 \%$ \\
\hline Total & 6,36 & 5,38 & $-0,97$ & $85 \%$ \\
\hline
\end{tabular}

Principales deficiencias en la calidad en el servicio de los micronegocios de artesanías de madera

En la Figura 1, se muestra los resultados de las expectativas y percepciones de la calidad en el servicio según las cinco dimensiones de cada instrumento aplicado y que los clientes de los micronegocios de artesanías de madera evaluaron, donde la dimensión de empatía obtuvo la mayor brecha con $-1,167$ puntos de diferencia entre expectativas y percepciones por lo que existe una gran insatisfacción por parte de los clientes, esto es a causa de que en los micronegocios no se respetan los horarios de trabajo, los empleados no se esmeran porque los clientes se sientan totalmente atendidos, satisfechos y porque no comprenden sus necesidades.

La segunda dimensión con la brecha más amplia es la de elementos tangibles con una puntuación de $-1,018$ esto es a consecuencia de que en los micronegocios no cuentan con equipos modernos como son los estantes y vitrinas para las artesanías de madera haciendo que no se vean atractivos para los clientes, por otra parte la fachada se encuentran deterioradas, no tienen áreas limpias y ordenadas, los empleados no tienen una apariencia limpia, ya 


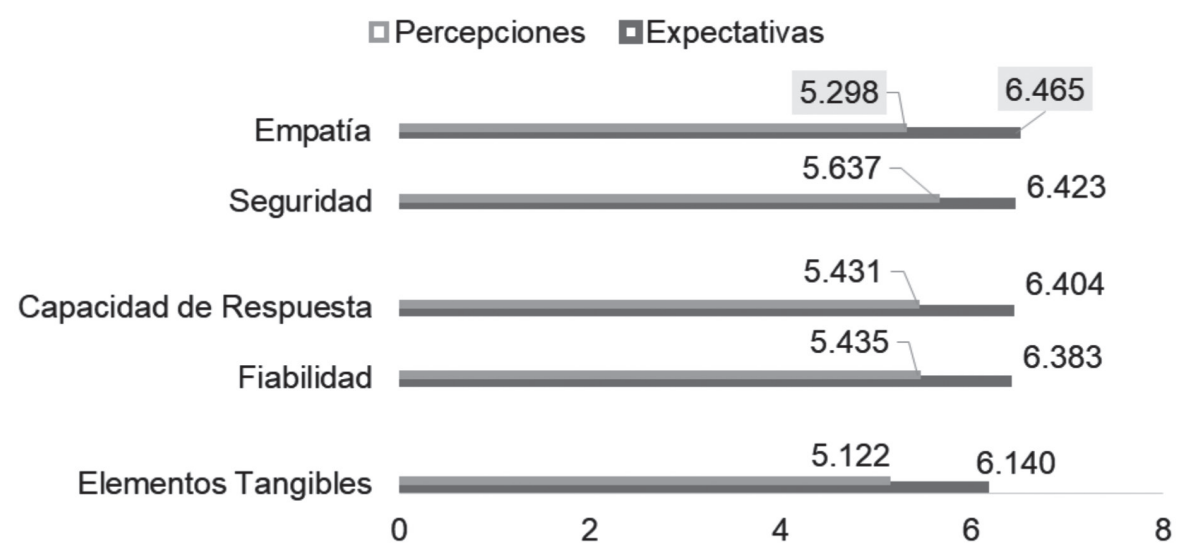

Figura 1. Expectativas y percepciones de las cinco dimensiones de la calidad en el servicio en los micronegocios de artesanías de madera.

que no están presentables en el momento de recibir a los clientes y al interior de las tiendas de artesanías no están bien iluminadas y ventiladas.

La dimensión de capacidad de respuesta es la tercera con la brecha entre expectativas y percepciones de $-0,974$ puntos debido a que los clientes no reciben una atención rápida, los empleados no responden a las preguntas debido a que no tienen información sobre los productos. La cuarta dimensión de fiabilidad con $-0,949$ puntos es a causa de que los empleados se equivocan al momento de atender a los clientes y por no cumplir en fecha y hora establecida para la entrega de productos, la falta de información de la ubicación y horarios de los micronegocios.

La última dimensión de seguridad es la que obtuvo la menor brecha de $-0,787$ puntos de calificación asignada por los clientes, ya que algunos se sintieron en confianza y en algunos micronegocios los empleados dan un trato amable y personalizado a sus clientes.

\section{CONCLUSIONES}

A continuación se expondrán las principales conclusiones a las que se llegó después de haber desarrollado la investigación respecto a la calidad en el servicio de micronegocios del sector artesanal de madera en una comisaría de Mérida, Yucatán.

En la dimensión de empatía donde las expectativas obtuvo 6,46 puntos y en percepciones 5,30 puntos con brecha de $-1,167$ puntos por lo que hay insatisfacción de parte de los clientes en la calidad en el servicio de los micronegocios de artesanías de madera, es a causa de que los micronegocios no respetaban los horarios de trabajo, los empleados no se esmeraban para que los clientes se sintieran atendidos y satisfechos, o no se preocupaban de que haya lo que los clientes buscaban, y los precios de los productos son elevados en algunos micronegocios. Es necesario que se respeten los horarios y que los empleados se esmeren por atender a los clientes, para que estos regresen.

En la dimensión de elementos tangibles existe una brecha de $-1,018$ puntos entre expectativas y percepciones debido a que en los micronegocios no cuentan con equipos modernos, áreas limpias y ordenadas, las fachadas están deterioradas, los trabajadores no están presentables y no cuentan con buena iluminación y ventilación adecuadas, por lo que reparar todo esto, no requiere de grandes cantidades de dinero. En la mejora de esto contribuirá a la imagen del micronegocio y a que los clientes vean atractivo el lugar y las artesanías de madera, es una manera de poder atraer la atención de nuevos clientes.

La dimensión de capacidad de respuesta obtuvo una brecha de $-0,974$ debido a que los clientes no estaban informados sobre cuándo surtirán productos que en ese momento no tienen los micronegocios, no recibían respuestas de parte de los empleados cuando se encontraban ocupados al igual que no les transmitían confianza, por lo que una propuesta de mejora es que los empleados se encuentren 
capacitados e informados sobre el funcionamiento del micronegocio, para dar una correcta información a los clientes si quedar en malos términos con ellos.

En la dimensión de fiabilidad se obtuvo una brecha de $-0,949$, debido a que los empleados no siempre solucionan problemas de los clientes respecto a información sobre la ubicación del micronegocio o los precios que tienen las artesanías de madera, al igual que en las entregas en fecha y hora prometida de los productos a los clientes. En esta dimensión se da una recomendación similar a la dimensión capacidad de respuesta, es importante que los dueños y los empleados estén informados sobre cambios que pudiera haber en el micronegocio de artesanía.

En la última dimensión de seguridad la brecha fue de $-0,787$ por lo que hay una pequeña insatisfacción por parte de los clientes debido a que los empleados en algunas ocasiones no eran amables con los clientes en el momento de atenderlos al igual que no recibían interés en sus necesidades. Es necesario que los empleados sean amables, que entiendan las necesidades del cliente, que los comprendan y que una buena actitud es beneficiosa para todos.

Todo este estudio contribuye a que los dueños de los micronegocios de artesanías de madera de la comisaría, tenga un contexto actual del servicio que brindan a sus clientes y puedan observar con los datos cuál es la expectativa que tienen y cuál es la percepción que obtienen los clientes al recurrir a su micronegocio, siendo así la manera de poder optimizar y mejorar sus servicios con las propuestas ya mencionadas, ya que estas no necesitan de grandes inversiones por parte de los dueños.

Mejorar en las cinco dimensiones: elementos tangibles, fiabilidad, capacidad de respuesta, seguridad y empatía; es una manera de atraer mayores clientes, ganarse su confianza y fidelidad, para así ser una herramienta que ayude a los micronegocios a permanecer en el mercado y ser competitivos ante su competencia, además de contribuir a la economía local.

Una de las recomendaciones para los dueños de los micronegocios de artesanías de madera en la comisaría de Dzityá, Yucatán es la capacitación para los empleados y los mismos dueños para ofrecer un servicio de calidad para los clientes, debido a que es importante la información y capacitación para el mejoramiento de los micronegocios como lo son las estrategias de ventas, el manejo del paquete office, higiene y seguridad entre otros, ya que esto les permitirá ganar la fidelidad de los clientes para poder permanecer en el mercado y poder adquirir una ventaja competitiva.

Cabe aclarar que las recomendaciones establecidas para mejorar la calidad en el servicio, no requieren grandes inversiones de dinero, por otra parte sólo se necesita disposición y tiempo de parte de los empleados y dueños de los micronegocios, así como una buena actitud al momento de tratar a sus clientes. En cuanto a los dueños, se debe procurar mantener una buena comunicación con el personal de contacto directo con los clientes para que se puedan sentir seguros de platicar puntos de interés o de mejoras en la forma de cómo brindar un mejor servicio, puesto que son ellos los que atienden todos los días a los clientes.

Es recomendable también, aplicar con frecuencia herramientas como son encuestas de salida, entrevistas a los clientes, grupos de enfoques, buzón de sugerencias, entre otras, con el fin de conocer mejor las necesidades de los clientes y así realizar mejoras continuas.

Esta investigación podrá servir para enriquecer los conocimientos teóricos y empíricos respecto a la calidad en el servicio, micronegocios de Dzityá y de las artesanías de madera, también podrá servir de referencia para futuras investigaciones.

\section{REFERENCIAS}

[1] V. Zeithmal, A. Parasuraman y L. Berry. "Calidad total en las gestión de servicios". Ediciones Díaz de Santos, S.A. Madrid. España. 1993. ISBN 978-84-7978-061-6.

[2] M. Martín y T. Suárez. "Impacto del capital humano y organizacional en las estrategias de calidad y servicio al cliente e innovacción de la PYME”. Academia de Ciencias Administrativas AC. Vol. $21 \mathrm{~N}^{\mathrm{o}} 35$, pp. 229-248. 2008.

[3] V. Hernández, D. Pineda y M. Andrade. "Las mipymes artesanales como un medio de desarrollo para los grupos rurales en México". Universidad \& Empresa. Vol. 13 $\mathrm{N}^{\circ}$ 21, pp. 65-92. 2011. 
[4] L. Schiffman y L. Lazar. "Comportamiento del consumidor". Pearson. Decima edición. México. 2010. ISBN 978-607-442-969-5.

[5] A. Santamaría y W. Cadrazco. "Matriz de inteligencia hotelera - mih. Una propuesta para el mejoramiento de la calidad en la prestación del servicio hotelero". Revista Pensamiento \& Gestión. № 31, pp. 211-246. 2011. ISSN 1657-6276.

[6] M. Pizzo. "Cómo servir con excelencia". Editorial académica española. Primera edición. España. 2012. ISBN 978-3-8484-5726-7.

[7] P. Larrea. "Calidad en el servicio del marketing a la estrategia". Editorial Díaz de Santos S. A. Primera edición Madrid, España. 1991. ISBN 84-7978-001-0.

[8] L. Berry, D. Bennet y C. Brown. "Calidad en el servicio: Una ventaja estratégica para instituciones financieras". Editorial Díaz de Santos. Cuarta Edición. Madrid, España. 1989. ISBN 978-848-718-925-8

[9] Centro de Investigación de mercados. "La importancia de un excelente servicio al cliente". Actualizado en enero de 2018. Fecha de consulta: 18 de febrero de 2018. URL: http://www.ciminvestigacion.com/ la-importancia-de-un-excelente-servicioal-cliente/

[10] M.V. Moya. "Estrategia: calidad de servicio". Revista Logistec supply chain managment y más. Actualizado el 21 de abril de 2016. Fecha de consulta: 2 de Marzo de 2018. URL: http://www.revistalogistec. com/index.php/scm/estrategia-logistica/ item/2278-estrategia-calidad-de-servicio

[11] A.F. Ramírez. "Calidad del servicio en los restaurantes de comida rápida en Puerto Ordaz, Venezuela. Caso: Makumba PizzaCafé". Revista Strategos. Vol. $5 \mathrm{~N}^{\circ} 10$, pp. 45-61. 2013.

[12] M. Torres y C.L. Vásquez. "Modelos de evaluación de la calidad del servicio; caracterización y análisis". Revista Compendium. Vol. 18 No 35, pp. 57-76. 2015.

[13] S. Maldonado, A. Guillén y R. Carranza. "Factores determinantes de la calidad del servicio de una cafetería en el campus de una universidad pública". Revista internacional administración y finanzas. Vol. $6 \mathrm{~N}^{\mathrm{o}} 1$, pp. 109-118. 2013.
[14] J.M. Daza. "Análisis de la medición de calidad en los servicios hoteleros". Revista Criterio libre. Vol. $11 \mathrm{~N}^{\circ}$ 19, pp. 260-280. 2013.

[15] B. Castillejos. "Análisis de la calidad del servicio en hoteles resort bahía de Huatulco, Oaxaca". Tesis para obtar el grado de maestro. Universidad Veracruzana. Huatulco, Oaxaca. 2009.

[16] J. Varela, R. Prat, C. Voces y A. Rial. "Una nueva escala para la evaluación de la calidad de los servicios de Hostelería". Revista Psicothema. Vol. 18 N $^{\circ}$ 1, pp. 135-142. 2006.

[17] Aiteco Consultores, SL. "El modelo Servqual de calidad de servicio". Actualizado en 2016. Fecha de consulta: 02 de Marzo de 2018. URL: https://www.aiteco.com/ modelo-servqual-de-calidad-de-servicio/

[18] J. Wigodski. “¿Qué es SERVQUAL?”. Revista Medwave. Vol. $3 \mathrm{~N}^{\circ}$ 10. 2003.

[19] J. Pérez y M. Merino. "Definicion de: Definición de micro empresa". Actualizado en 2009. Fecha de consulta: 20 de Febrero de 2018. URL: http://definicion.de/ micro-empresa/

[20] P. Peña, I. Ríos y S. Salazar. "Los micronegocios en México: Razones para emprenderlos, expectativas, tamaño y financiamiento". Revista Estudios Económicos CNBV. Vol. 1, pp. 75-94. 2012.

[21] INEGI. "Censos económicos 2014: micro, pequeña, mediana y gran empresa, estratificación de los estalecimiento". Editorial INEGI. México. 2014.

[22] INEGI. "Encuesta Nacional sobre Productividad y Competitividad de las Micro, Pequeñas y Medianas Empresas (ENAPROCE)". Editorial INEGI. México. 2015.

[23] C. Carriado. "Pymes mexicanas y su estrategia para 2017'. Forbes. Actualizado el 11 de Abril de 2017. Fecha de consulta: 7 de Marzo del 2018. URL: https://www.forbes.com.mx/ pymes-mexicanas-y-su-estrategia-para-2017/

[24] INEGI. "Información por entidad. Ciudad de México". Actualizado en 2015. Fecha de consulta: Enero de 2018. URL: http:// cuentame.inegi.org.mx/monografias/ informacion/df/poblacion/

[25] SEFOE. "Dirección de Planeación y Análisis Económicos de la SEFOE con datos del INEGI". Censo económico 2014. Yucatán. 2014. 
[26] O. Aquileo. "Casa de artesanías en Mérida, Yucatán”. Actualizado en Abril de 2016. Fecha de consulta: 10 de Febrero de 2018. URL: https://en-yucatan.com.mx/ merida-yucatan/casa-de-las-artesania/

[27] Yucatán Gobierno del Estado. (2012). "Cultura y tradiciones". Atualizado en 2012. Fecha de consulta: 10 de Febrero de 2018. URL: http://www.yucatan.gob.mx/?p=artesanias

[28] Yucatán Today. "XVI Edición de la Feria Artesanal Diztyá Tunich 2017”. Actualizado en Agosto de 2017. Fecha de consulta: 12 de Febrero de 2018. URL: http://yucatantoday. com/feria-artesanal-tunich-2017/
[29] O. Aquileo y J. Domínguez. "Dzityá". Actualizado en 2004. Fecha de consulta: 14 de Febrero de 2018. URL: https://en-yucatan. com.mx/merida-yucatan/dzitya/

[30] M. Guatzozón, A.M. Canto y H.A. Ulibarri. "La calidad en el servicio y su impacto en la permanencia de los micronegocios: propuesta de estudio de caso Dzityá, Yucatán”. ACACIA. pp. 66-77. 2017. ISBN 978-607-8153-43-5.

[31] J. Castañeda, M. Torre, J. Morán y L. Lara. "Metodología de la investigación". McGraw-Hill Interamericana Editores. México. 2002 